\title{
Research Paper \\ Strength and Range of Motion of Internal and External Rotator Muscles in Vol- leyball Players With and Without Uneven Shoulders
}

\author{
${ }^{*}$ Abdolrasoul Daneshjoo ${ }^{1}$ (D) Tavoos Hosseini $^{2}$
}

1. Department of Sport Biomechanics and Corrective Exercise, East Tehran Branch, Islamic Azad University, Tehran, Iran.

2. Department of Physical Education, East Tehran Branch, Islamic Azad University, Tehran, Iran.

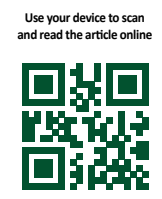

Cittation: Daneshjoo A \& Hosseini T. [Strength and Range of Motion of Internal and External Rotator Muscles in Volleyball Players With and Without Uneven Shoulders (Persian)]. Journal of Sport Biomechanics. 2019; 5(3):134-145. https://doi. org/10.32598/biomechanics.5.3.1

https://doi.org/10.32598/biomechanics.5.3.1

Key words:

Strength, Internal rotation, External rotation, Even shoulders, Uneven shoulders

\section{ABSTRACT}

Objective Performing repetitive overhead movements puts stress on the shoulder joint, leading to uneven shoulders and pain in the shoulder joint. The purpose of this study was to evaluate and compare the strength and Range of Motion (ROM) of internal and external rotator muscles in volleyball players with and without uneven shoulders.

Methods This is a descriptive and causal-comparative study conducted on 30 volleyball players randomly assigned into two groups of patients with uneven shoulders and healthy peers. A dynamometer (Manual muscle testing) was used to measure the strength of the rotator muscles at zero degrees, a goniometer to measure the ROM of muscles, and a scoliometer device to measure the symmetry of shoulders.

Results There was a significant difference in the strength of internal $(P=0.03)$ and external $(P=0.02)$ rotator muscles between groups with and without uneven shoulders. The ROM of internal $(P=0.02)$ and external $(P=0.03)$ rotator muscles were also significantly different between the two groups.

Conclusion High muscle strength in volleyball players with uneven shoulders is a risk factor. Limitations in the ROM of rotator muscles in the shoulder joint of these athletes showed the need for more attention to preventive approaches by creating corrective strategies.

\section{Extended Abstract}

\section{Introduction}

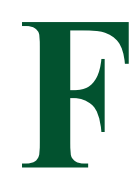

or having favorable physical condition, coordination of different parts of the body is required. One of the areas that play a key role in maintaining good physical condition is the scapula. The role of the scapula has been significantly considered in recent years, as it is very important in the production of smooth and coordinated movements of the shoulder girdle. Kibler [2] stated that the role of the scapula in throwing and serving is to create the right movements and positions for facilitating shoulder function. In other words, proper shoulder movements are critical for natural shoulder mechanics and are effective during throwing balls. The position of the scapula and its orientation are clearly different in throwing and non- throwing athletes, which indicates that adaptations in these athletes depend on their field of activity [4]. The inability of the scapula to perform these roles results in loss of physiological and biomechanical efficiency, and, therefore, causes shoulder dysfunction. This can lead to poor performance and increased shoulder injuries [5].

\section{* Corresponding Author:}

Abdolrasoul Daneshjoo.

Address: Department of Sport Biomechanics and Corrective Exercise, East Tehran Branch, Islamic Azad University, Tehran, Iran.

Tel: +98 (912) 2061034

E-mail: phdanesh@yahoo.com 
One of the main roles of the scapula is that it acts as a base for connecting muscles, and its displacement can affect the function of the muscles around the shoulder girdle, especially the muscles that support the scapula. In addition, the position of the scapula is directly related to the production of muscle forces as well as the range of motion of the shoulder girdle [5]. Therefore, it seems that displacement of the scapula may affect the function and production of the muscle force in the shoulder girdle, especially the scapular stabilizer muscles. In volleyball athletes, repetitive movements such as abduction and external rotation are accompanied by extension and internal rotation, and hitting the ball at maximal abduction increases the traction force. Muscle imbalances and rotation muscles weakness are risk factors for the shoulder injury in overhead athletes [6]. Regarding the main cause of changes in the distance of the scapula from the spine, some researchers have stated that the position of the scapula is an inherited condition that some people are born with [7]. However, others believe that if the muscles that support the scapula do not have the necessary endurance, strength, and flexibility, there will be many changes in this area, given the important role they play in the position of the scapulae relative to each other.

The literature review showed that very little research has been done on the strength and range of motion of the muscles around the shoulder, as well as the ratio of strength between the muscles around the glenohumeral joint in elite athletes, and most of them have examined the external/internal rotation ratio in shoulder. On the other hand, some studies have mainly compared the physical strength of female and male athletes or athletic and non-athletic women and have generally concluded that male athletes have more physical strength than female athletes, or female athletes are in a better position than non-athletic women in terms of physical strength or other physical fitness factors. Therefore, due to the fact that the balance of strength between the agonist and antagonist muscles of the glenohumeral joint can cause damage to the shoulder joint and impingement syndrome, it is necessary to conduct research in this area. To our knowledge, no study has been conducted in this area, and none of them have examined the effect of the position of the scapula on the strength of the shoulder girdle muscles in elite athletes. In this regard, this study aimed to assess and compare the strength and Range of Motion (ROM) of external and internal rotation of shoulder muscles in male volleyball players

\section{Methods}

The study population consists of all male volleyball players in Mahabad County with at least 3 years of regular exercise experience and having practice at least 5 sessions per week. Of them, 15 with uneven shoulders and 15 healthy peers were randomly selected as the statistical sample of this study and entered the study after obtaining written consent from them. A Manual Muscle Testing (MMT) dynamometer $(\mathrm{CI}=95 \%)$ was used to measure the isometric strength of the shoulder muscles. Strength of internal and external rotators was measured in a neutral position (zero degrees of abduction). The ROM of the internal and external rotation of the glenohumeral joint in one of the shoulders was evaluated twice a day with a standard goniometer (Universal, LTD Co., Japan) with an accuracy of 3 degrees. To measure uneven shoulders, a scoliometer (Mizuho OSI, Japan) with acceptable validity and reliability.

Table 1. Independent t-test and ANOVA results of comparing internal and external rotation strength and ROM of two study groups

\begin{tabular}{|c|c|c|c|c|c|}
\hline \multirow{2}{*}{ Variables } & \multicolumn{2}{|c|}{ Mean \pm SD } & \multirow{2}{*}{$\mathbf{t}$} & \multirow{2}{*}{$\mathbf{f}$} & \multirow{2}{*}{$\mathbf{P}$} \\
\hline & Affected & Healthy & & & \\
\hline Internal rotation strength of dominant side & $16.32 \pm 4$ & $13.17 \pm 3.1$ & -3.87 & 9.109 & 0.03 \\
\hline Internal rotation strength of non-dominant side & $13.75 \pm 3.7$ & $14.40 \pm 3.2$ & 98 & 0.94 & 0.35 \\
\hline External rotation strength of dominant side & $2.7 \pm 14.55$ & $11.32 \pm 2.3$ & -3.77 & 10.46 & 0.02 \\
\hline External rotation strength of non-dominant side & $3.5 \pm 11.41$ & $12.23 \pm 2.7$ & 94 & 0.89 & 0.45 \\
\hline Internal rotation ROM of dominant side & $72.15 \pm 8.2$ & $82.5 \pm 7.4$ & -5.77 & 8.166 & 0.03 \\
\hline Internal rotation ROM of non-dominant side & $82.91 \pm 7.5$ & $85.58 \pm 6.4$ & 90 & 0.97 & 0.41 \\
\hline External rotation ROM of dominant side & $107.10 \pm 6.1$ & $100.9 \pm 7.02$ & -6.87 & 11.043 & 0.02 \\
\hline External rotation ROM of non-dominant side & $101.8 \pm 7.9$ & $98.45 \pm 5.1$ & 71 & 0.74 & 0.10 \\
\hline
\end{tabular}


The collected data were analyzed in SPSS V. 20 using descriptive (mean, standard deviation) and inferential statistics. Independent t-test was used to compare the strength and ROM of even and uneven shoulders, and paired t-test and ANOVA were used to compare two study groups. The normality of data distribution was performed by Kolmogorov-Smirnov test due to the sample size $>30$. The significance level was set as $\mathrm{P}<0.05$.

\section{Results}

The results shown in Table 1 showed that there was a significant difference in internal $(\mathrm{P}=0.03)$ and external rotation strength $(\mathrm{P}=0.02)$ of volleyball players with and without uneven shoulders. A significant difference was also reported in their internal $(\mathrm{P}=0.02)$ and external rotation $\mathrm{ROM}(\mathrm{P}=0.03)$.

\section{Conclusion}

The high muscle strength of volleyball players with uneven shoulders has been identified as a risk factor in many studies. In volleyball, it can also be considered a long-term cause of injury. Volleyball, like other sports, involves repetitive movement patterns in which hands have activity overhead, and in the long term, it can lead to the asymmetry of shoulder on the dominant side which can cause muscle imbalance and limitation in the ROM of rotator muscles in the shoulder joint of these athletes. It requires attention to preventive approaches by creating corrective solutions. It is recommended that volleyball coaches and players, given the specific biomechanics of repetitive volleyball movements and to prevent imbalances in muscle strength and ROM, emphasize equally in strengthening and stretching the agonist and antagonist muscles of the shoulder girdle, and when exercising, pay attention to the muscles that are undergoing strengthening or flexibility exercises.

\section{Ethical Considerations}

Compliance with ethical guidelines

This article is taken from Research plan in Physical Education and Sport Sciences Lab. All subjects voluntarily participated in the study and signed the written consent form. IR. IAUETB.98001 Article Code of Ethics

Funding

This research did not receive any specific grant from funding agencies in the public, commercial, or not-forprofit sectors.

\section{Authors' contributions}

All authors contributed in preparing this article.

\section{Conflicts of interest}

The authors declared no conflict of interest. 


\title{
مطالعه قدرت و دامنه حركتى بازوى واليباليستهاى داراى شانه متقارن و نامتقارن
}

\author{
'عبدالرسول دانشجو' 0. طاووس حسينى' \\ ا.كروه بيومكانيك و حركات اصلاحي، واحد تهران شرق، دانشعاه آزاد اسلامى، تهران، ايران.

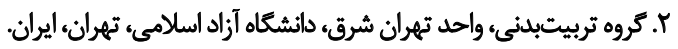

\begin{abstract}
حكيد
هدف اجراى حركات تكرارى و ضربات بالاي سر، مفصل شائه را تحت استرس قرار ميى دهد و سبب ايجاد شائه نامتقارن و درد در مفصل

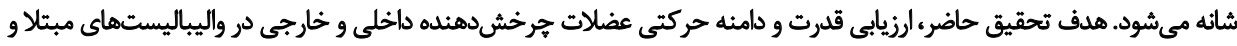

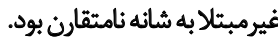

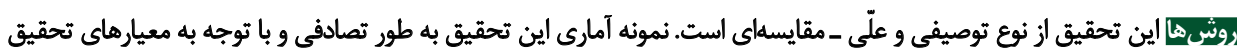

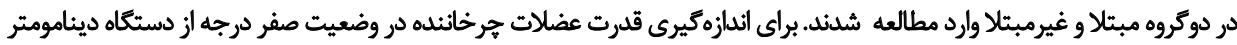

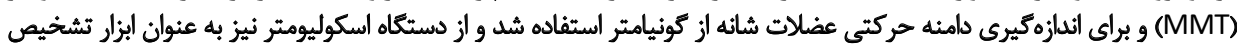

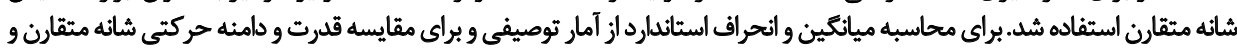

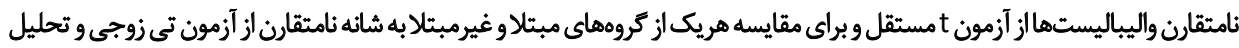

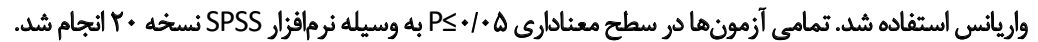

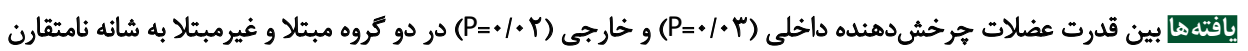

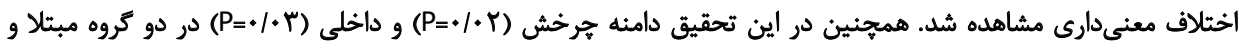

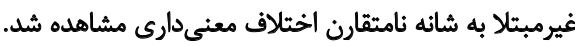

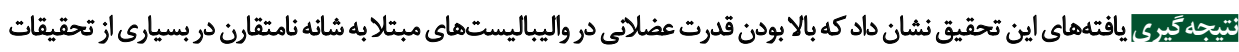

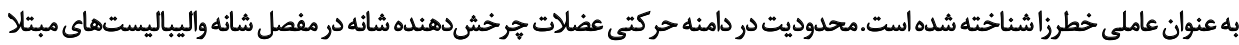

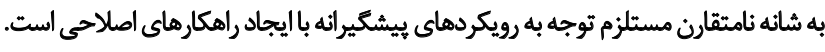

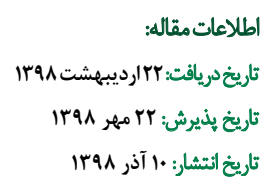

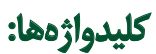

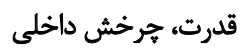
بازو، جرخش خانه مارجي بازو، شانه مثقارن، شانه نامتثقارن

يرتابي، تفاوت مشخصى باورزشكاران غيرير تابيى دارد كه نشان دهنده

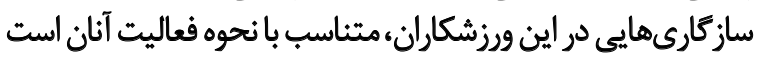

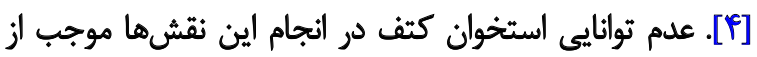

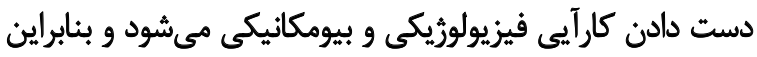

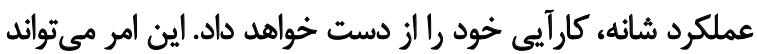

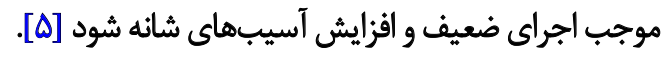

ازجمله نقشهاى اصلى استخوان كتف اين است كه به عنوان

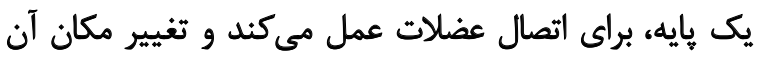

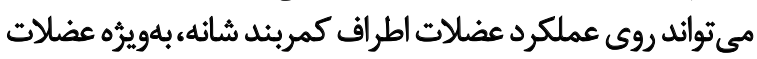

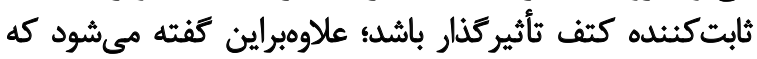

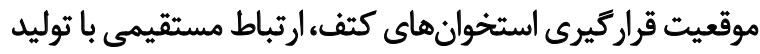

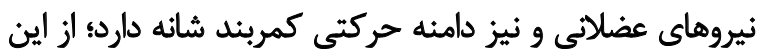

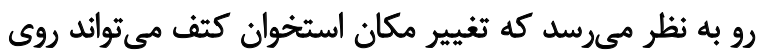

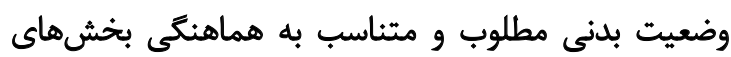

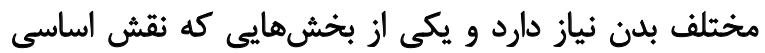

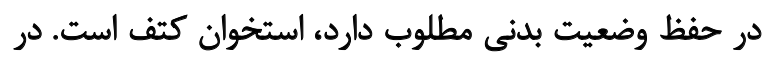

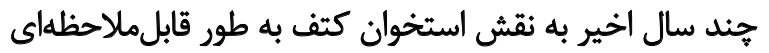

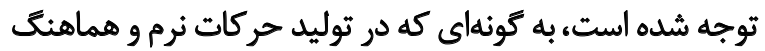

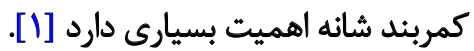

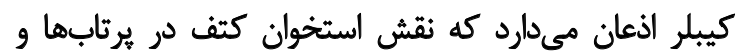

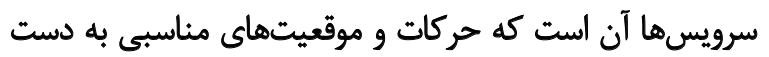

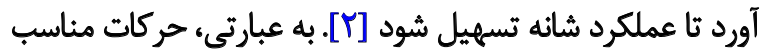

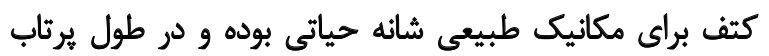

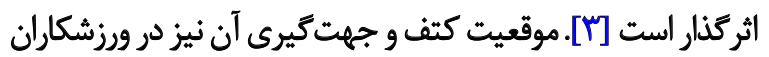




\section{روششناسى}

يُرؤهش حاضر از نوع تحقيقات توصيفى-على-مقايسهاى

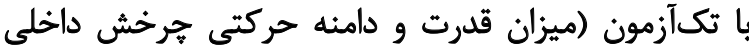

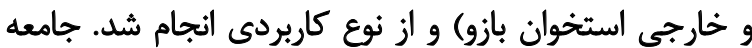

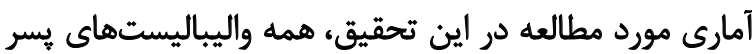

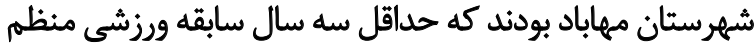

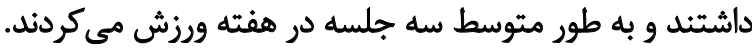

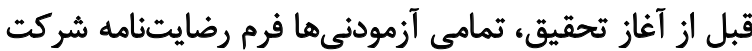

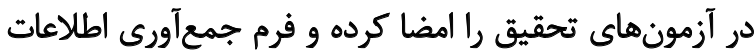

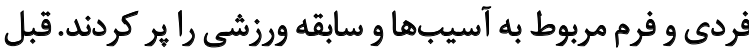

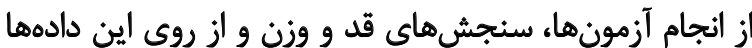

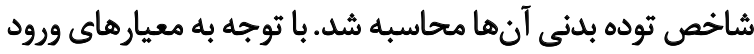

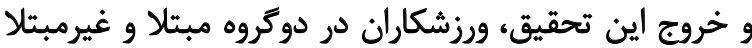

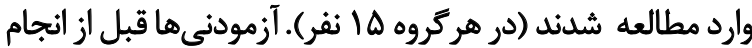

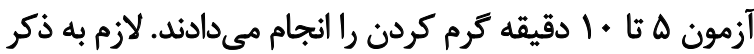

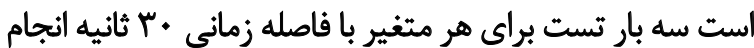

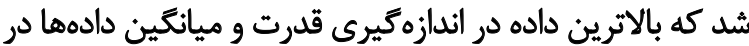
دامنه حركتي مبناى كار آمارى قرار تركت فيرت

ابتدا به وسيله اسكوليومتر درجه نامتقارن بودن شانهها

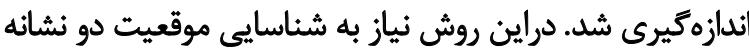

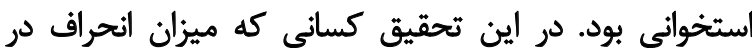

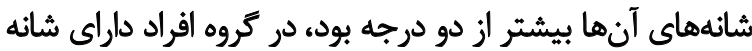

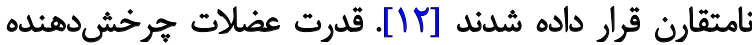

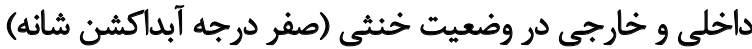

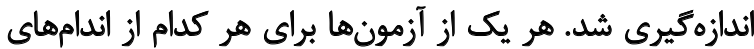

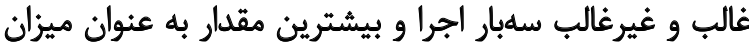

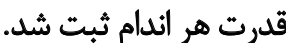

براى ارزيابى دامنه جرخش به خارج، در شرايطى كه آزمودنى

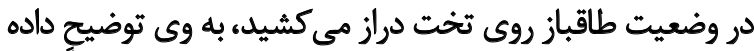

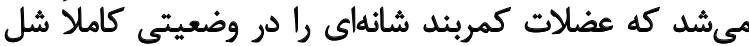

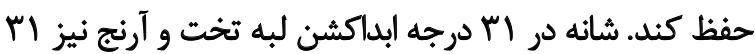

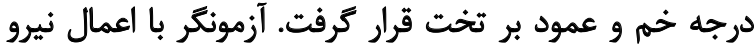

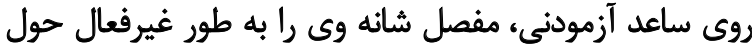

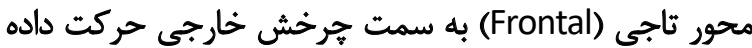

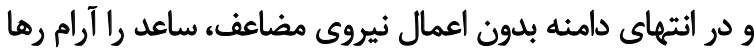

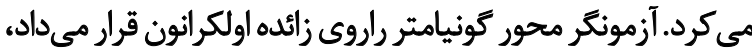

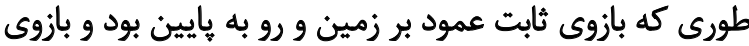

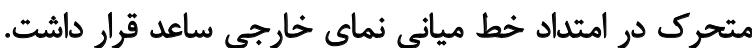

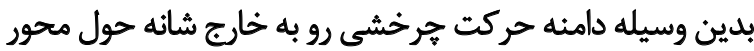

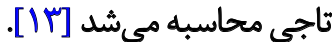

براي ارزيابى دامنه جرخش به داخل در شرايطى كه آزمودنى

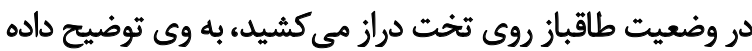

عملكرد و توليد نيروى عضلاتي كمربند شانه، بلويره عضلات

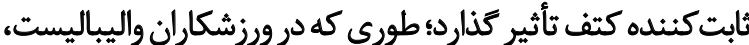

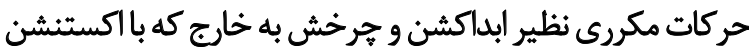

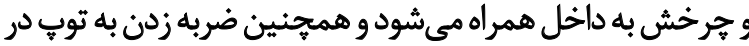

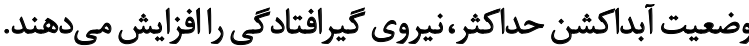

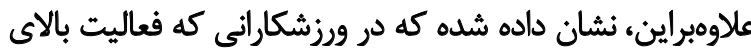

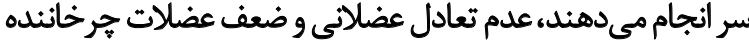

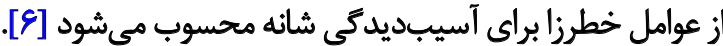
برخى محققان در مورد علت اصلى تغييرات فاصله استخوانهاى

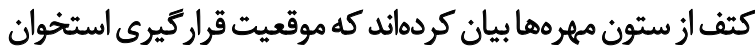

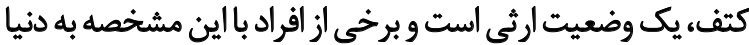

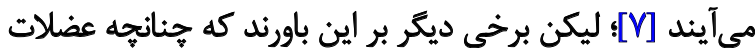

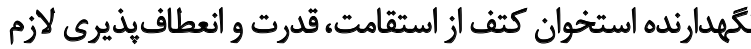

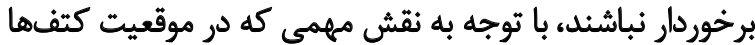

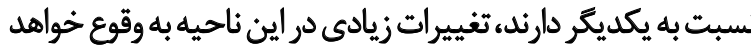

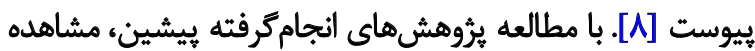

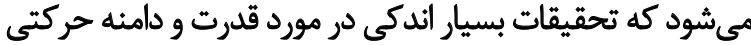

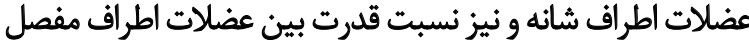

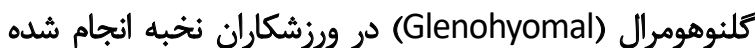

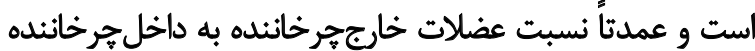

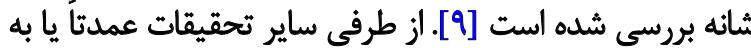

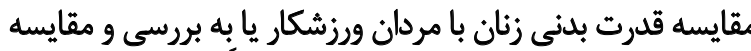

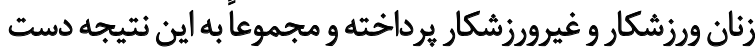

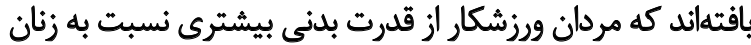

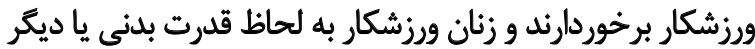

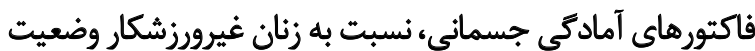

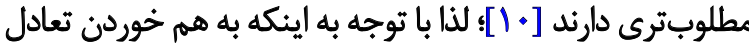

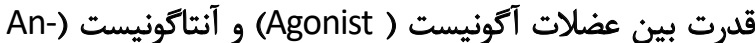
مفصل كلنوهيومرال مى تواند موجب صدمات (tagonist

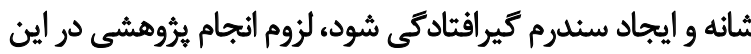

خصوص خودنمايي مى كند [11].

ازآنجاكه محقق در اين خصوص و در مورد ورزشكاران به

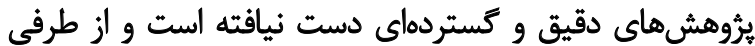

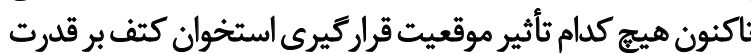

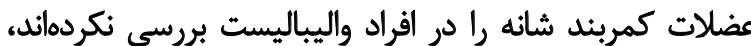

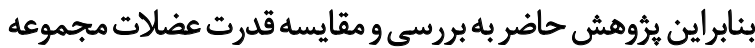

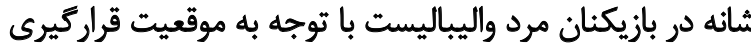

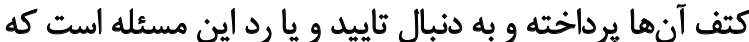

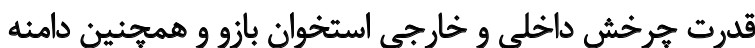
حركتى در خرخش داخلى وختى خارئى شانه متقارن و نامتقارن اختلاف معنادارى دارئ دارد. 
از تروههاي مبتلا و غيرمبتلا به شائه نامثقارن از آزمون t زوجى و

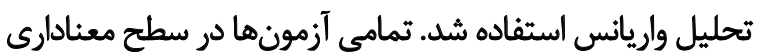

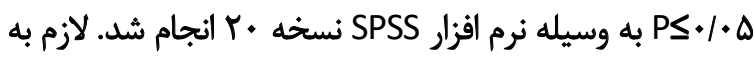

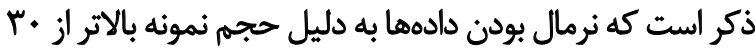
نفر با آزمون كولموكروف اسميرونف انجام ترفت

ثtن

در جدول شماره ا ميانكين و انحراف استاندارد سن، قد و ومئن

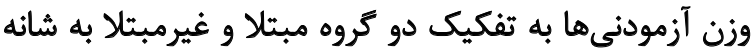
ن امتقارن ارائه شده است.

جدول شماره ب نتايج آزمون تى مستقل و تحليل واريانس را

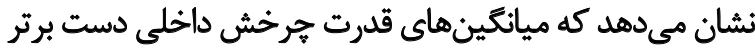

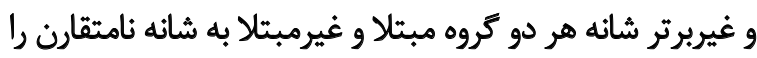

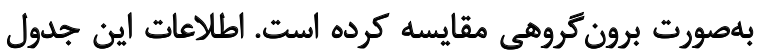

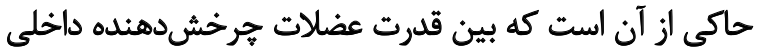

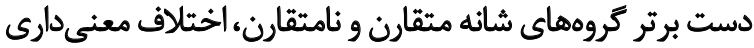

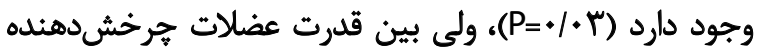

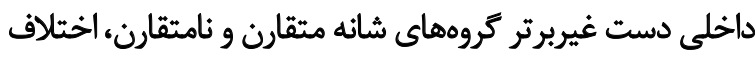

$$
\text { معنى دارى مشاهده نمي شئرد. }
$$

جدول شماره ب نتايج آزمون تى جفتى را نشان مى دهد كه

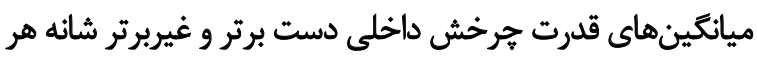

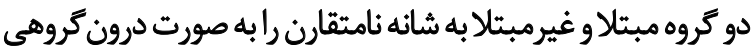

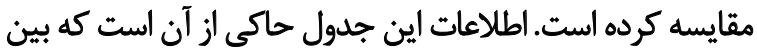

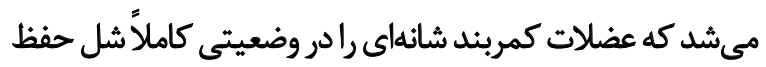

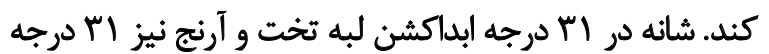

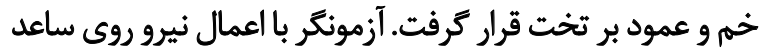

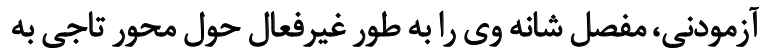

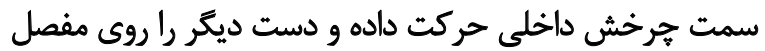

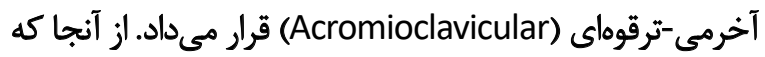

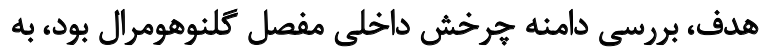

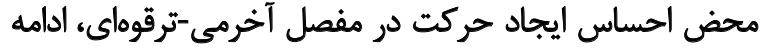

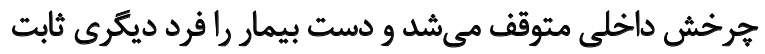

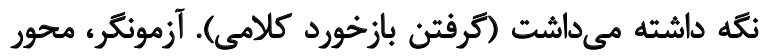

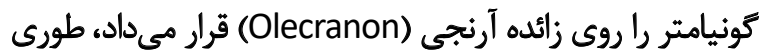

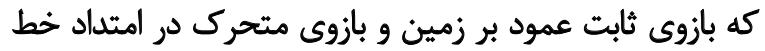

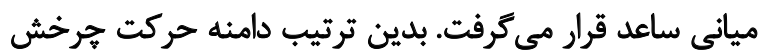

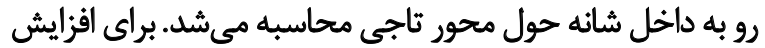

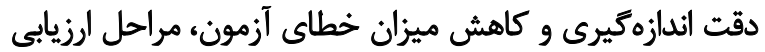

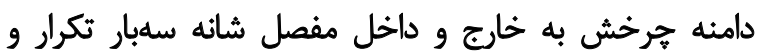

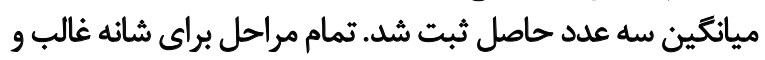

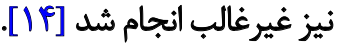
تجزيه و تحليل ياقتهها

براي تجزيه و تحليل اطلاعات بهدستآمده از آمار توصيفى و

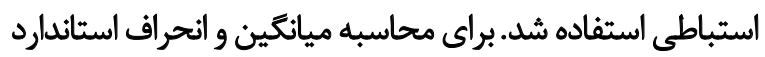

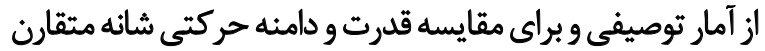

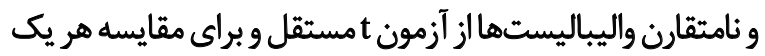

جدول ا. شاخصهاى آنترويومتريك افراد شركتكئنده در دو كروه

\begin{tabular}{|c|c|c|c|c|}
\hline وزن (كيلوكرم) & قد (ساتتىمتر) & سن (سال) & تعداد & كروه \\
\hline$V T / v \pm r / 1$ & $\mid V \varepsilon / f \pm f / \mu$ & $r \mu \pm 1 / t$ & 10 & شانه نامتقارن \\
\hline$V^{e} / T \pm Y / f$ & $|W / r \pm r /|$ & $r \Psi \pm T h$ & 10 & شانه مثقارن \\
\hline
\end{tabular}

مجله بيومكانيك وزنش

جدول ب. نتايج آزمون تى مستقل و تحليل واريائس مقايسه قدرت جرخش داخلى كروههاى مبتلاو غيرمبتلا

\begin{tabular}{|c|c|c|c|c|c|}
\hline \multirow{2}{*}{ نمره P P } & \multirow{2}{*}{ نمره f } & \multirow{2}{*}{ نمره تى } & \multicolumn{2}{|c|}{ مياتكين ثانحراف معيار } & \multirow{2}{*}{ كروه } \\
\hline & & & كروه غيرمبتلا & كئروه هبثلا & \\
\hline $.1+r$ & $9 / 1.9$ & $-r / A V$ & $m / / v_{ \pm} m / n$ & $\mid \& / T_{ \pm}{ }^{p}$ & قدرت هرخش داخلي دست برتر \\
\hline ه &.$/ 97$ & $u$ & $\| f / \mu= \pm T / r$ & $I r / N \Delta_{ \pm} r / N$ & قدرت هرخش داخلى دست غيربرتر \\
\hline
\end{tabular}

جدول r. نتايج آزمون تى جفتى قدرت جرخش داره داخلى دست برتر و غيربرتر

\begin{tabular}{|c|c|c|c|c|}
\hline Pمره P & نمره تى & دست غيربرتر & دست برتر & كروه \\
\hline$+1++f$ & $r / 4$ & $I r / N \Delta_{ \pm} r / V$ & $1 E / T_{ \pm}{ }^{f}$ & قدرت جرخش داخلى مبتلا \\
\hline I.r & $V / r$ & $\| f / x \cdot \pm r / r$ & $\left|r / \Lambda Y_{ \pm} r /\right|$ & قدرت جرخش داخلى غيرمبتلا \\
\hline
\end{tabular}


جدول f. نتايج آزمون تى مستقل و تحليل واريانس مقايسه قدرت جرخش خارجى گروههاى مبتلا و غيرمبتلا

\begin{tabular}{|c|c|c|c|c|c|}
\hline \multirow{2}{*}{ Pمره P P } & \multirow{2}{*}{ نمره f } & \multirow{2}{*}{ نمره تى } & \multicolumn{2}{|c|}{ 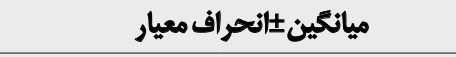 } & \multirow{2}{*}{ كروه } \\
\hline & & & كروه غير مبثلا & كروه مبتلا & \\
\hline $.1 . r$ & $1 . / 48$ & $-r / W$ & $11 / M T \pm Y / T$ & $r / N \pm I F / \Delta \Delta$ & قدرت هرخش خارجى دست برتز \\
\hline.$/ 10$ &.$/ 19$ & 94 & $I T / \pi T \pm T / N$ & $r / \Delta \pm \mid 1 / p)$ & قدرت هرخش خارجى دست غيربرتر \\
\hline
\end{tabular}

جدول هـ نتايج آزمون تى جفتى قدرت جرخش خارجى دست برتر و غيربرتر

\begin{tabular}{|c|c|c|c|c|}
\hline \multirow{2}{*}{ ت نمره P } & \multirow{2}{*}{ تمره تى } & \multicolumn{2}{|c|}{ 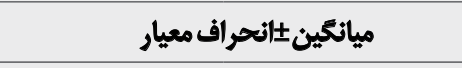 } & \multirow{2}{*}{ كروه } \\
\hline & & دست غيريرتر & دست برتر & \\
\hline $.1 .+$ & $-M / M$ & $\mid W / F i \pm r / \Delta$ & $I F / \Delta \Delta \pm T / V$ & قدرت جرخش خارجى مبتلا \\
\hline.$/ 4 a$ & Af & $\mid r / T M \pm r / Y$ & $\mathbb{W} / \mathrm{T} \pm T / T$ & قلرت جرخش خارجى غيرمبتلا \\
\hline
\end{tabular}

جدول \&. نتايج آزمون تى مستقل و تحليل واريانس مثايسه دامنه حركتى جرخش داخلى كروههاى مبتلاو غيرمبتلا

\begin{tabular}{|c|c|c|c|c|c|}
\hline \multirow{2}{*}{ 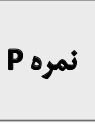 } & \multirow{2}{*}{ نمره } & \multirow{2}{*}{ 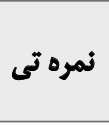 } & \multicolumn{2}{|c|}{ ميانكين土|نحراف معيار } & \multirow{2}{*}{ كروه } \\
\hline & & & كروه غيرمبئلا & كئروه مبثلا & \\
\hline.$/ . r$ & NIES & $-\Delta / W$ & $A T / \Delta \pm V / F$ & $r / 1 \Delta \pm N T$ & دامنه حركتى جرخش داخلى دست برثر \\
\hline.$/ 41$ &.$/ 9 V$ & 9. & $\Lambda \Delta / \Delta \Lambda \pm \& / \uparrow$ & $V / \Delta \pm \lambda Y / q)$ & دامنه حركتى جرخش داخلى دست غيربوتر \\
\hline
\end{tabular}

جدول V. نتايج آزمون t جفتى دامنه حركتى جرخش دانس داخلى دست برتر و غيربرتر

\begin{tabular}{|c|c|c|c|c|}
\hline \multirow{2}{*}{ Pمره P } & \multirow{2}{*}{ تمره T T } & \multicolumn{2}{|c|}{ ميانكين土|نحراف معيار } & \multirow{2}{*}{ كروه } \\
\hline & & دست غيربرتر & دست برتر & \\
\hline $.1+r$ & $-9 / W$ & $A r / q) \pm Y / \Delta$ & $R / 1 \Delta \pm N / Y$ & دامنه حركتى هرخش داخلى مبتلا \\
\hline .10. & $1+1$ & $\Lambda \Delta / \Delta \Lambda \pm E / F$ & $A T / T \Delta \pm V / \varphi$ & دامنه حركتى جرخش داخلى غيرمبتلا \\
\hline
\end{tabular}

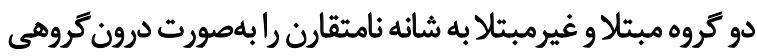

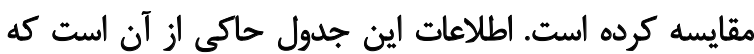

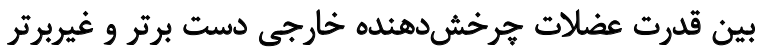

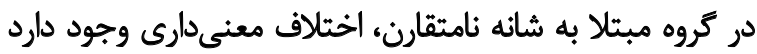

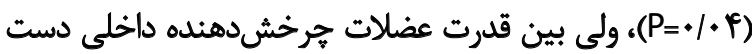

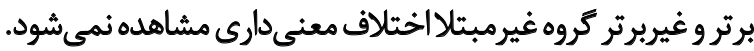
جدول شماره ع نتايج آزمون تي مستقل و تحليل واريانس

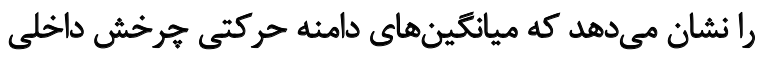

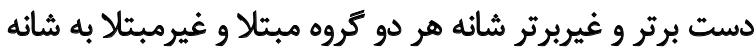

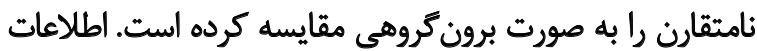

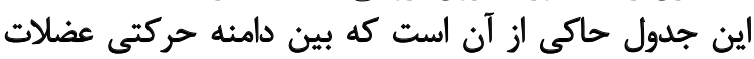

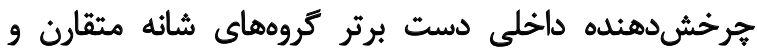

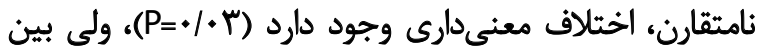

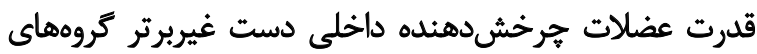

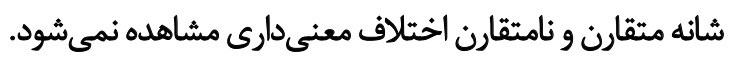

قدرت عضلات جرخش دهنده داخلي دست برتر و غيربرتر در كروه

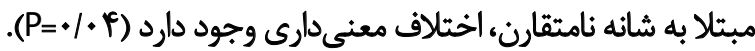

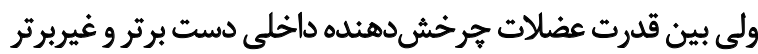

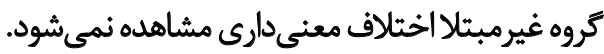

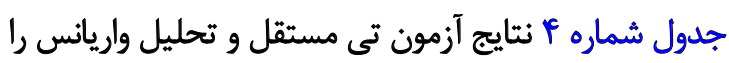

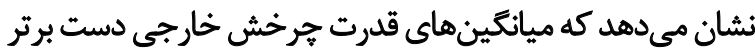

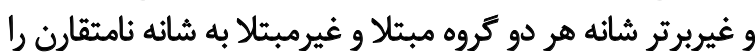

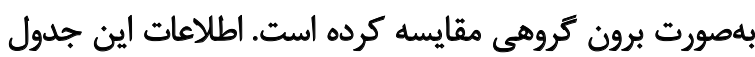

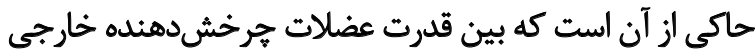

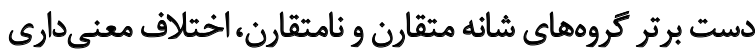

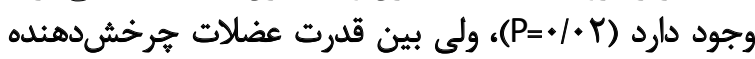

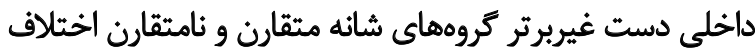
معنى دارى مشاهله نميى داستود.

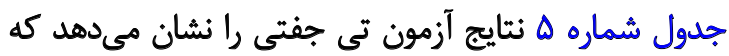

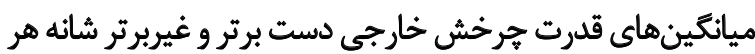


جدول A. نتايج آزمون تى مستقل و تحليل واريانس دامنه حركتى جرخش خارجى گروههاى مبتلا و غيرمبتلا

\begin{tabular}{cccccc}
\hline & & \multicolumn{2}{c}{} & \\
\cline { 4 - 6 } \\
\hline
\end{tabular}

جدول 1. نتايج آزمون تى جفتى دامنه حركتى هرخش خارجى دست برتر و غيربرتر

\begin{tabular}{|c|c|c|c|c|}
\hline \multirow{2}{*}{ نمره P } & \multirow{2}{*}{ نمره تى } & \multicolumn{2}{|c|}{ ميانكين土|نحراف معيار } & \multirow{2}{*}{ كيووه } \\
\hline & & دست غيربرتر & دست برتر & \\
\hline $1+f$ & $-I Q / A \varphi$ & $1 \cdot 1 / A \pm V / 9$ & $1 \cdot V / 1 \cdot \pm \& /$ & دامنه حركتى هرخش خارجى مبثلا \\
\hline.$/ 2 \mu$ & so & $Q / F \Delta \pm \Delta / 1$ & $1 \cdot \cdot / q \pm V / \cdot r$ & دامنه حركتي خرخش خارجي دست غيرمبنالا \\
\hline
\end{tabular}

مجله بيومكانيك وزنش

جرخش شهنده داخلى دست برثر دو زروه مبتلا و غيرمبتلا به

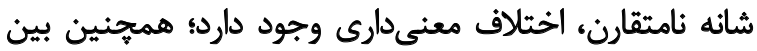

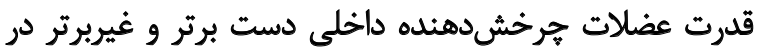

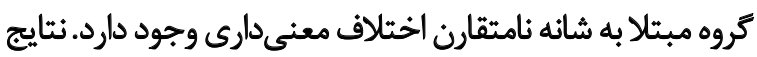

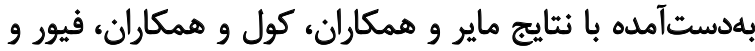

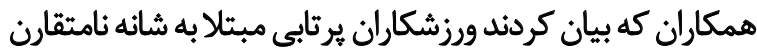
داراى قدرت بيشترى هستئد، همخواني دارد [س]؛؛ ولى بي با نتايج

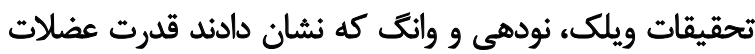

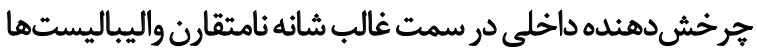

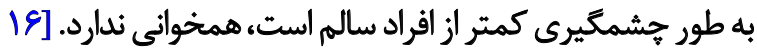

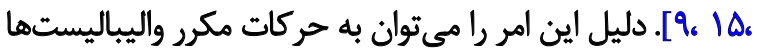

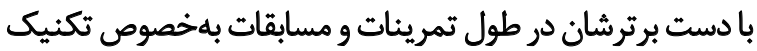

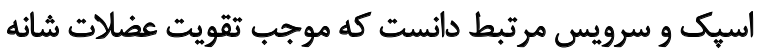

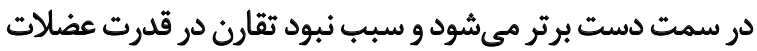

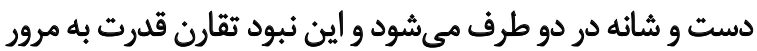
زمان به افزايش نبود تقارن در شانهها نيز مي دئ دانجامد. عمل يرتاب يا ضربه خصوصاً در سطح بالا مستلزم اين است كه

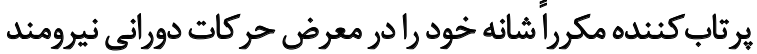
و تكرارى قرار دهد. اين تكرارحركات منجر به آموزش عصبه

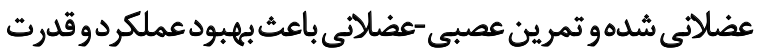

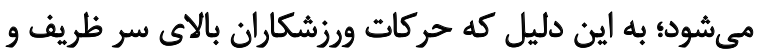

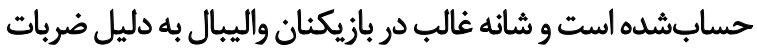

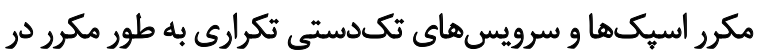

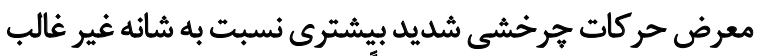

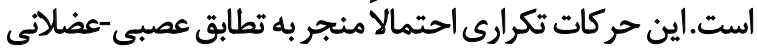

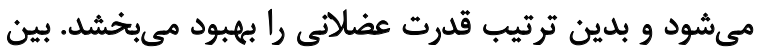

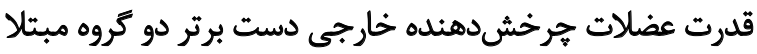

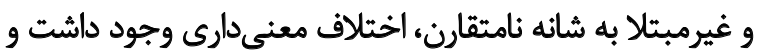

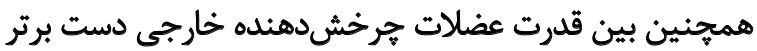

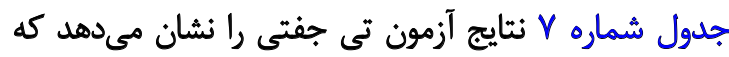

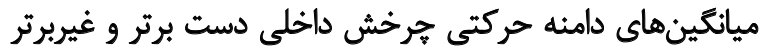

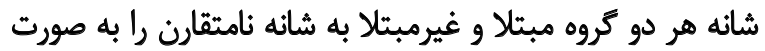

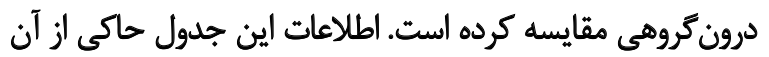

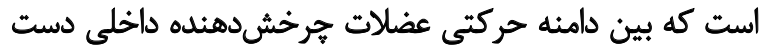

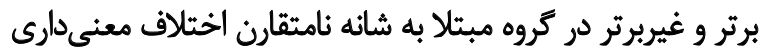

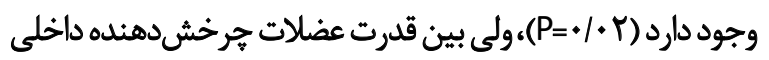

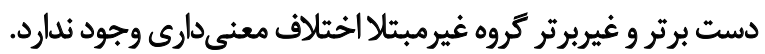
جدول شماره A نتايج آزمون تى مستقل و تحليل واريانس را

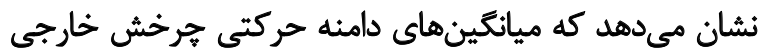

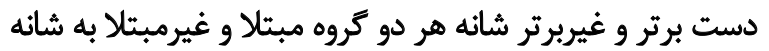

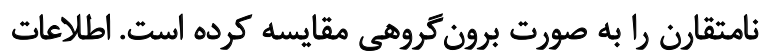

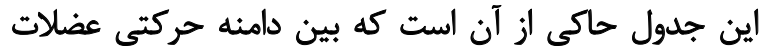

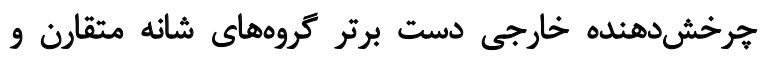

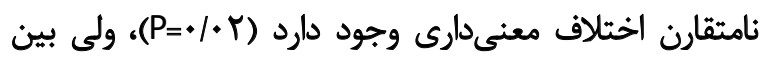

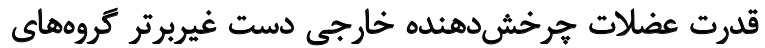

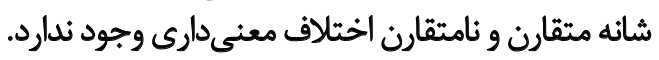
جدول شماره 9 نتايج آزمون تي جفتى را نشان ميدهد كه

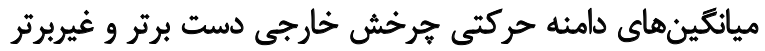

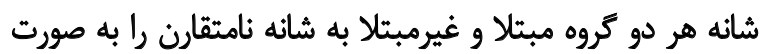

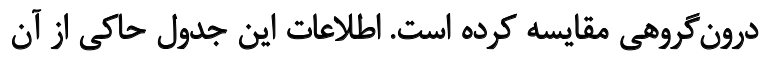

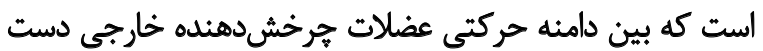

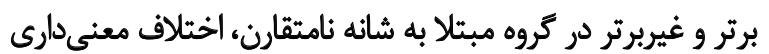

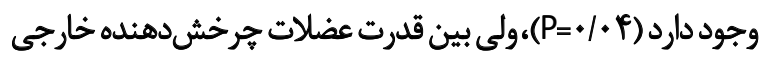

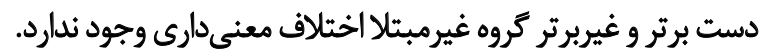

ب

يافتههاي تحقيق حاضر نشان داد كه بين قدرت عضلات 


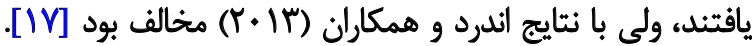

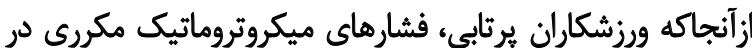

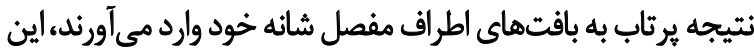

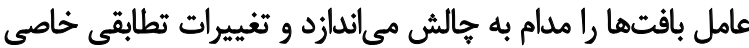

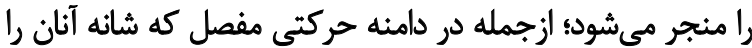

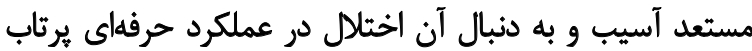

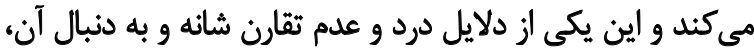

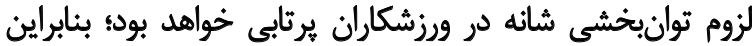

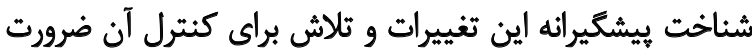

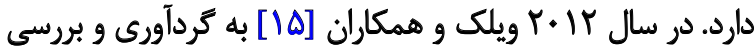

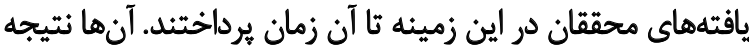

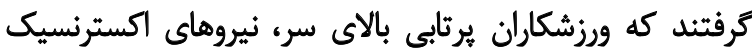

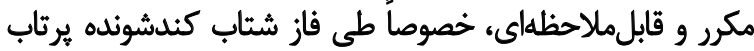

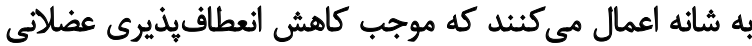

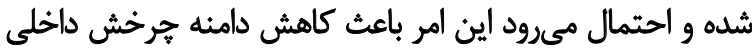

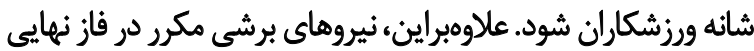

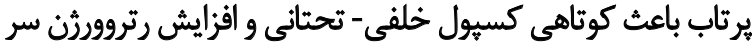

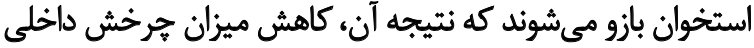

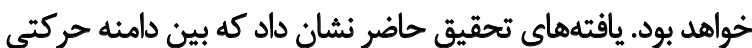

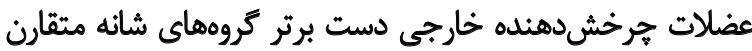

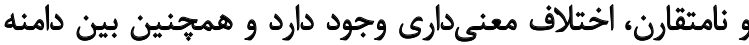

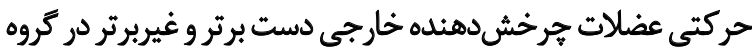

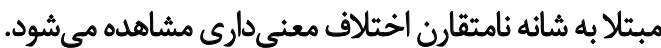

بر اساس نتايج بهدستآمده در اين تحقيق مشاهده شد كه

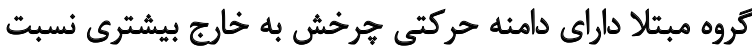

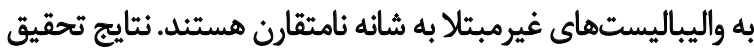

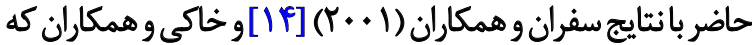

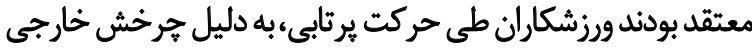

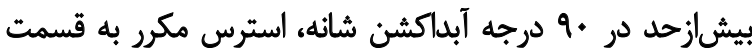

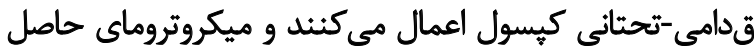

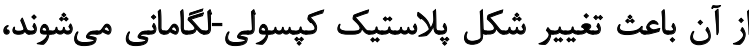

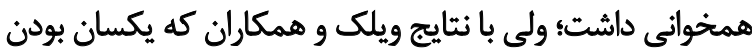

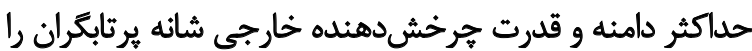

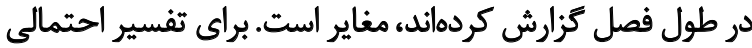

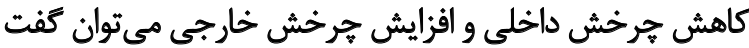

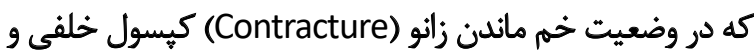

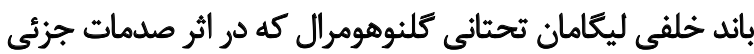
مكرر در طى فاز شتاب منفى ير إثاب ايجاد

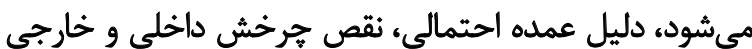

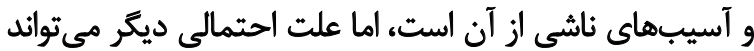

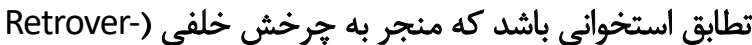

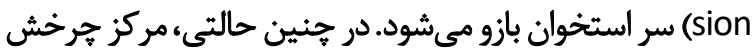

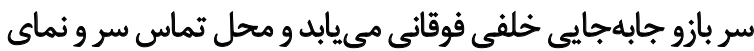

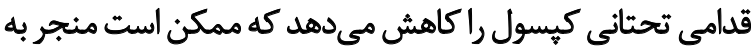

و غيربرتر در كروه مبتلا به شانه نامتقارن اختلاف معنى دارى

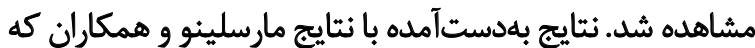

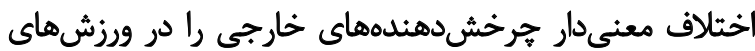
يكسطرفه تأييد كردند، موافق است، ولى باني

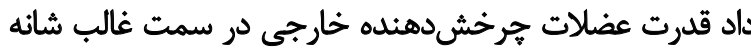

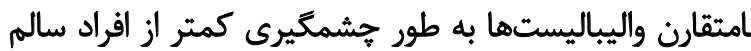

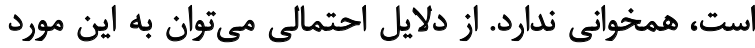

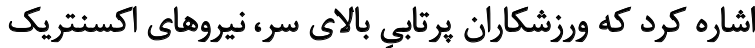

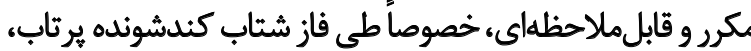

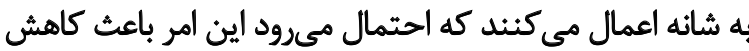

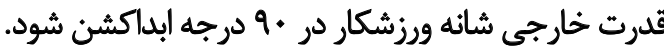
عضلات جرخش دهنده به عنوان يايين كشندهاو ثبات دهندهن

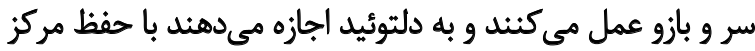

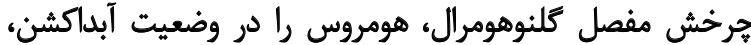

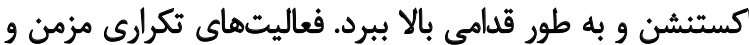

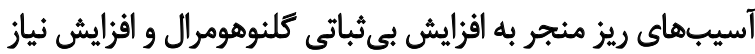

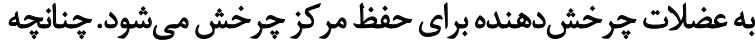

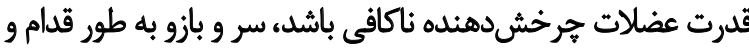

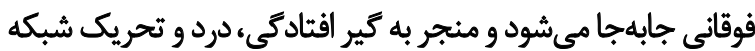

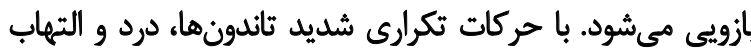

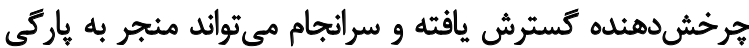

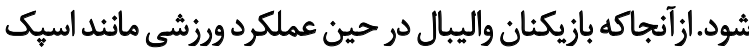

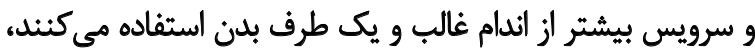

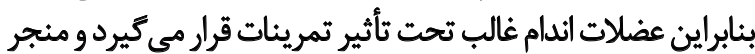

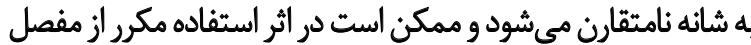

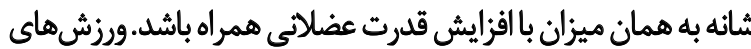

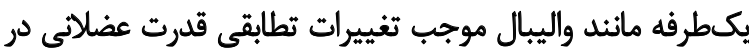

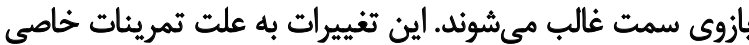

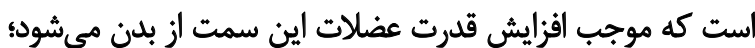

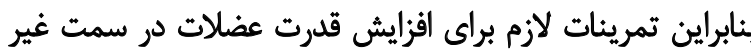

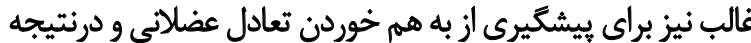
أسيبديدگى ضرورى به نظر مىرسد.

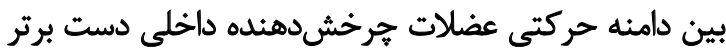

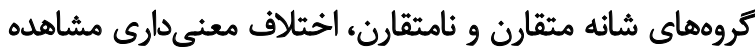

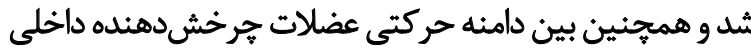

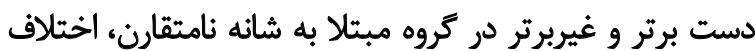

$$
\text { معنى دارى مشاهده شد. }
$$

بر اساس نتايج اين تحقيق، مشاهده شد كه كروه مبتلا داراى

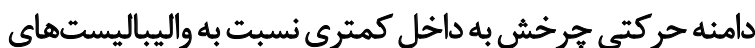

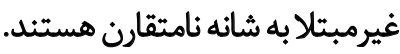

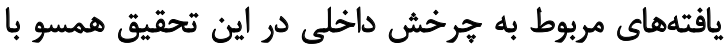

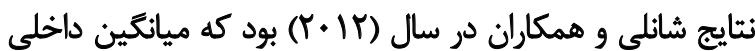

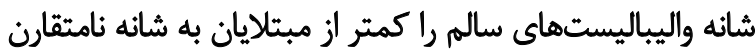




\section{ملاحظات اخلاقى \\ ييروى از اصول اخلاق يثوهش}

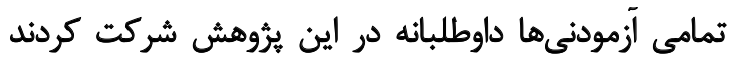

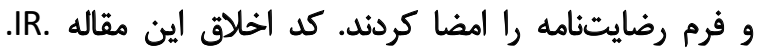

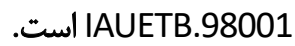

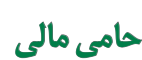

$$
\begin{aligned}
& \text { اين مقاله حامى مالى نداشته است. } \\
& \text { مشاركت ثنويسند انَّان } \\
& \text { تمامى نويسندكان در آمادهازى اين يثرهش مشاركت } \\
& \text { داشتهاند. }
\end{aligned}
$$

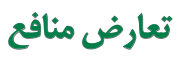

بنابر اظهار نويسندكان اين مقاله تعارض منافع ندارد.
تفاوتهاى دامنه حركتى شانه متقارن و نامتقارن واليباليستها

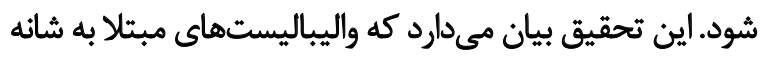

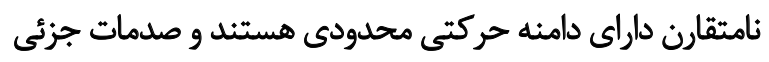

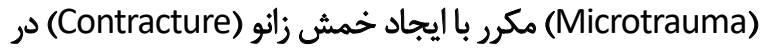

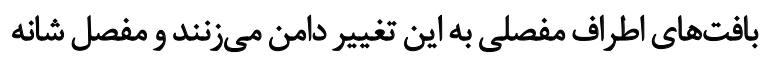

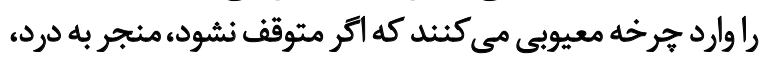

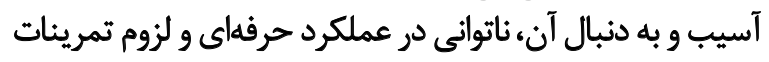

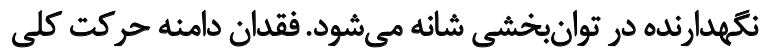

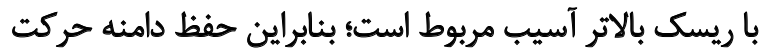

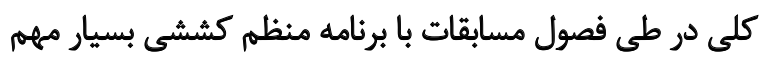

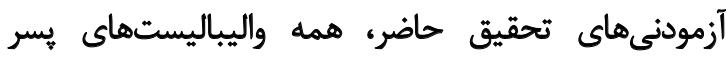

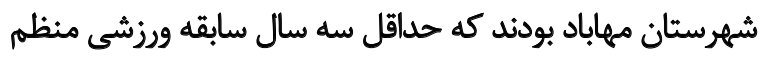

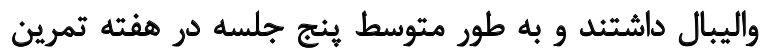

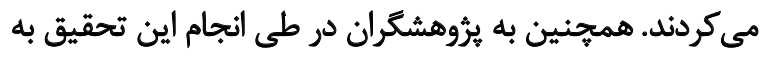

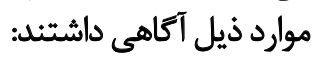

سطح علاقه و ميزان رغبت آزمودنىها به شركت در تحقيق

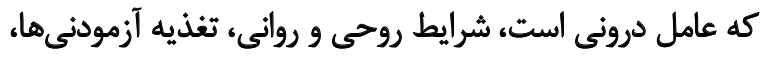

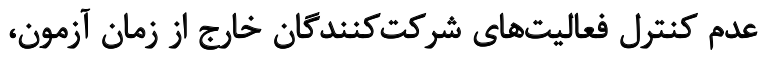

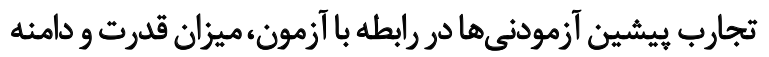
حركتى جرخش دهندهائ شائه اوليه افراد.

\section{نتيجليَيرى نههايى}

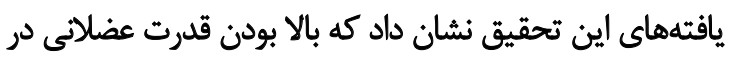

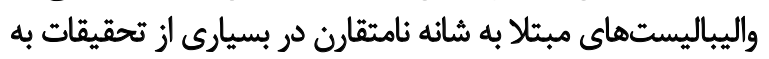

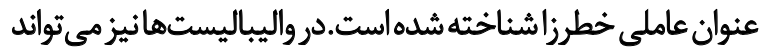

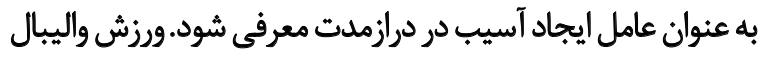

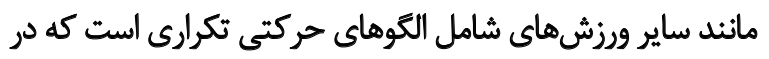

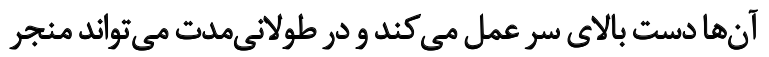

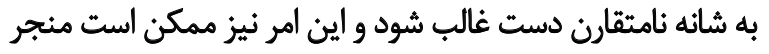

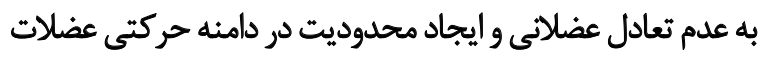

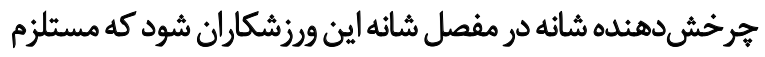

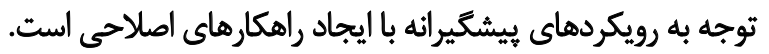

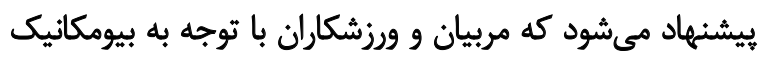

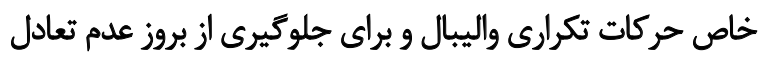

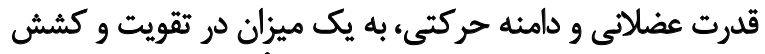

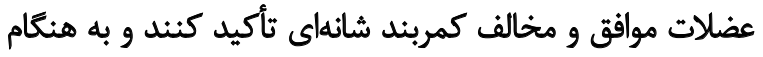

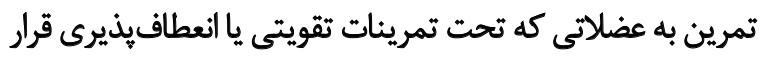
مى ميرند، توجه داشته باشند. 


\section{References}

[1] Thomson BC, Michell RS. The effects of repetitive exercise of the shoulder on lateral scapular stability. Presented at: American therapy association combined sections meeting, 18 jun 2012. New Orleans, LA.

[2] Kibler WB. Scapular disorder. In: Garrete WE, Speer KP. Principles Practice of Orthopedic Sports Medicine, Lippincott, Kirkendall DT, Williams \& Wilkins. 2000, 27: 497-510.

[3] Meyer KE, Seather EE, Soiney EK, Shebeek MS, Paddock KL, Ludewig PM. Three-dimensional Scapular Kinematics during the throwing motion. J Appl Biomech. 2008; 24(1):24-34. [DOI:10.1123/jab.24.1.24] [PMID]

[4] Myers JB, Laudner KG, Pasquale MR, Bradley JP, Lephart SM. Scapular position and orientation in throwing athletes. American J Sports Med. 2005; 33(2):263-71. [DOI:10.1177/0363546504268138] [PMID]

[5] Houglum PA. Therapeutic exercise for athletic injuries, Human Kinetics. 2000; 11:342-69.

[6] Kibler WB. The role of the scapula in athletic shoulder function. Am J Sports Med. 1998; 6(2):325-37. [DOI:10.1177/0363546598026002280 1] [PMID]

[7] Mottram SI. Dynamic stability of the scapula. Man Ther. 1997; 2(3):12331. [DOI:10.1054/math.1997.0292] [PMID]

[8] Odom CJ, Taylor AB, Hurd CE, Denegar CR. Measurement of scapula asymmetry and assessment of shoulder disfunction using the lateral scapula slide test: A reliability and validity study. J Phys Ther. 2001; 81(2):800-9. [DOI:10.1093/ptj/81.2.799] [PMID]

[9] Wang HK, Cochrane T. Mobility impairment, muscle imbalance, muscle weakness, scapular asymmetry, and shoulder injury in elite volleyball athletes. J Sports Med Phys Fitness. 2001; 41(3):403-10. [PMID]

[10] Donatelli R, Ellenbecker TS, Ekedahl SR, Wilkes JS, Kocher K, Adam J. Assessment of shoulder strength in professional baseball pitchers. J Orthop Sports Phys Ther. 2000; 30(90):546-51. [DOI:10.2519/ jospt.2000.30.9.544] [PMID]

[11] Yildiz Y, Aydin T, Sekir U, Kiralp MZ, Hazneci B, Kalyon TA. Shoulder terminal range eccentric antagonist/concentric agonist strength ratios in overhead athletes. J Med Sci Sports. 2006; 16(3):174-80. [DOI:10.1111/ j.1600-0838.2005.00471.x] [PMID]

[12] Bonagamba GH, Coelho DM, Anamaria S, de Oliveira AS. Inter and intra-rater reliability of the scoliometer. Revista Brasileira de Fisioterapia. 2010; 14(5):432-8. [PMID]

[13] Seminati E, Minetti AE. Overuse in volleyball training/practice: A review on shoulder and spine-related injuries. Eur J Sport Sci. 2013; 13(6):732-43. [DOI:10.1080/17461391.2013.773090 ] [PMID]

[14] Safran MR, Borsa PA, Lephart SM, Fu F, et al. Shoulder proprioception in baseb all pitchers. J Shoulder Elbow Surg. 2001; 10(5):438-44. [DOI:10.1067/mse.2001.118004] [PMID]

[15] Wilk KE, Macrina LC, Fleisig GS, Porterfield R, Simpson CD, Herker P, etal. Correlation of Glenohumeral internal rotation deficit and total rotation motion to shoulder injuries in professional baseball pitchers. Am J Sports Med. 2011; 39(2):329-35. [DOI:10.1177/0363546510384223] [PMID]

[16] Nodehi Moaghadam A, Nasrin N, Kharazmi A, Eskandari Z. A comparative study on shoulder rotational strength, range of motion and proprioception between the throwing athletes and non-athletic persons. Asian
J Sports Med. 2013; 4(1): 34-40. [DOI:10.5812/asjsm.34528] [PMID] [PMCID]

[17] Andrade MS, Vancini RL, Lira CAD, Mascarin NC, Fachina RJ, Silva $A C D$. Shoulder isokinetic profile of male handball players of the Brazilian National Team. Braz J Phys Ther (BJPT). 2013; 17(6):572-8. [DOI:10.1590/S1413-35552012005000125] [PMID] [PMCID] 
